\title{
Turbulence and coarsening in active and passive binary mixtures
}

\author{
S. Berti ${ }^{1}$, G. Boffetta ${ }^{1}$, M. Cencini ${ }^{2,4}$, A. Vulpiani ${ }^{3,4}$ \\ 1 Dipartimento di Fisica Generale and INFN Università di Torino, Via Pietro Giuria 1, I-10125 Torino, Italy. \\ 2 ISC-CNR, Via dei Taurini 19, I-00185 Roma, Italy. \\ ${ }^{3}$ Dipartimento di Fisica and INFN Sezione Università di Roma "La Sapienza", P.zzle Aldo Moro 2, I-00185 Rome, Italy. \\ ${ }^{4}$ INFM-SMC P.zzle Aldo Moro 2, I-00185 Rome, Italy.
}

(Dated: November 5, 2018)

\begin{abstract}
Phase separation between two fluids in two-dimensions is investigated by means of Direct $\mathrm{Nu}-$ merical Simulations (DNS) of coupled Navier-Stokes and Cahn-Hilliard equations. We study the phase ordering process in the presence of an external stirring acting on the velocity field. For both active and passive mixtures we find that, for a sufficiently strong stirring, coarsening is arrested in a stationary dynamical state characterized by a continuous rupture and formation of finite domains. Coarsening arrest is shown to be independent of the chaotic or regular nature of the flow.

PACS numbers: 47.27.-i,05.70.Ln
\end{abstract}

When a binary fluid mixture at the critical concentration is cooled from a high temperature to a sufficiently low temperature (below a critical one), the original homogeneous phase becomes unstable and spontaneously evolves into two phases separated by an interface. As time advances an out-of-equilibrium process of phase ordering takes place through the formation of domains of a single phase that grow algebraically in time [1]. In fluids, the presence of a hydrodynamic velocity field makes this process more complicated than the corresponding one in solid alloys. For instance, since Siggia's seminal work [2], it is well known that hydrodynamics may accelerate the domain growth (see Refs. [3] and [4] for recent development in three and two dimensional fluids, respectively). Phase ordering dynamics becomes even more complex and less understood when the fluid mixture is externally driven $[5,6]$; beyond their theoretical interest, phase separating binary fluids under flow embody a great technological interest [7] for their distinctive rheological properties. This problem has been extensively investigated in shear flows $[8,9,10,11]$ where coarsening becomes highly anisotropic: the single-phase domain growth accelerates in the shear direction while in the transversal one the growth is arrested [10,11] or strongly slowed down [9]. Less clear is the case in which the mixture is stirred by a turbulent flow $[7,12,13,14]$. Here, phase separation may be completely suppressed [13], or a dynamical steady state with domains of finite length and well defined phases may develop $[6,14]$. A similar phenomenology has been experimentally observed in stirred immiscible fluids [15]. This Letter aims to clarify the nature of the non-equilibrium steady state, characterized by the continuous rupture and formation of domains.

Previous investigations focused on passive binary mixtures (when the feedback of the phase ordering on fluid velocity is neglected) in random flows [16] and in chaotic flows [17] (in a Lagrangian sense, i.e. two initially very close particles separate exponentially in time [18]). Here we focus on the phase ordering dynamics of active two- dimensional binary mixtures in which the fluid is driven by chemical potential inhomogeneities [6]. By means of numerical experiments we show that coarsening arrest is a generic and robust phenomenon, whose existence can be understood by an energy conservation argument. Moreover, we show that in the passive limit Lagrangian Chaos is not necessary for coarsening arrest.

In the presence of stirring, the main question concerns the competition between thermodynamic forces, driving the phase segregation, and fluid motion, leading to mixing and domains break-up. For very high flow intensities phase separation can be completely suppressed [12, 13] due to mixing of the components and inhibition of interface formation. In active mixtures with very low viscosities such a phenomenon may be self-induced by the feedback [4, 19]: the fluid responds vigorously to local chemical potential variations and re-mixes the components. On the other hand, stirring may lower the critical temperature $[5,14,20]$. However, by performing a deeper quench, phase separation in a nontrivial statistically stationary state may still develop [6].

Being interested in deep quenching, here we work at zero temperature, as in Refs. $[16,17]$. We consider a symmetric $(50 \%-50 \%)$ mixture of two incompressible fluids of equal density $\rho=1$ and viscosity $\nu$. Such a bi-component system is described by a scalar order parameter $\theta(\boldsymbol{r}, t)$, the local fraction of the two fluids. The associated Landau-Ginzburg free energy reads [1]:

$$
\Phi[\theta]=\int \mathrm{d} \boldsymbol{r}\left(-\frac{1}{2} \theta^{2}+\frac{1}{4} \theta^{4}+\frac{\xi^{2}}{2}|\nabla \theta|^{2}\right),
$$

being $\xi$ the equilibrium correlation length, which provides a measure of the interface width. The dynamics is then governed by the Cahn-Hilliard $(\mathrm{CH})$ equation

$$
\partial_{t} \theta+\boldsymbol{v} \cdot \nabla \theta=\Gamma \nabla^{2} \frac{\delta \Phi}{\delta \theta}=\Gamma \nabla^{2} \mu
$$

where $\mu=-\theta+\theta^{3}-\xi^{2} \nabla^{2} \theta$ is the chemical potential, and $\Gamma$ is a mobility coefficient that we assume constant and independent of $\theta$. Hydrodynamics enters in Eq. (2) through 
the convective term. The order parameter is transported by the two-dimensional velocity field $\boldsymbol{v}$, which evolves according to the Navier-Stokes (NS) equation

$$
\partial_{t} \boldsymbol{v}+\boldsymbol{v} \cdot \boldsymbol{\nabla} \boldsymbol{v}=\nu \nabla^{2} \boldsymbol{v}-\nabla p-\theta \nabla \mu+\boldsymbol{f},
$$

where $p$ is the pressure. The fluid is forced by the external mechanical force $f$ and by local chemical potential variations $-\theta \nabla \mu$ (the two phases want to demix and thus force the fluid, see also Ref. [3] for a detailed derivation). This latter term can be rewritten as $-\xi^{2} \nabla^{2} \theta \nabla \theta$ plus a gradient term which can be absorbed into the pressure [6]. Therefore Eq.(3) formally reduces to the 2D magnetohydrodynamics (MHD) equation for the velocity field. Actually phase ordering and MHD share many phenomenological properties [6].

We numerically integrate the coupled equations (2)(3) by means of a standard pseudo-spectral code implemented on a two-dimensional periodic box of size $2 \pi \times 2 \pi$ with $512^{2}$ collocation points. Statistical analysis of domain sizes is obtained by considering the characteristic length, defined as $L(t)=\left\langle\left(1-\theta^{2}\right)\right\rangle^{-1}$ where $\langle\ldots\rangle$ denotes spatial average [21]. The initial condition for the order parameter is a high temperature configuration with $\theta$ set as white noise in space. In the presented results time is rescaled with the diffusive time $t_{m}=\xi^{2} / \Gamma$.

Unstirred case. Starting from the initial configuration with the fluid at rest $(\boldsymbol{v}=0)$, after a few diffusive time scales $t_{m}$, sharp interfaces appear and phase separation proceeds through domain coarsening. At long times, the domains length $L$ - the only characteristic scale of the system (provided $L \gg \xi$ ) - grows in time as a power law [1]. In 2D different regimes have been predicted and observed [4]: $L(t) \sim t^{1 / 3}$, as in fluids at rest, for high viscosity; $L(t) \sim t^{2 / 3}$ for lower viscosities. At intermediate values of viscosity it is still unclear whether there is only one characteristic scale [4]; for $\nu \ll 1$ and low mobility $\Gamma \ll 1$ mixing may overwhelm phase demixing [19]. In the following we will limit our analysis to the turbulent, low viscous, regime where scaling exponent $2 / 3$ is expected. This exponent can be dimensionally derived by balancing the inertial term $\boldsymbol{v} \cdot \boldsymbol{\nabla} \boldsymbol{v}$ with $\theta \boldsymbol{\nabla} \mu$ in (3), and assuming that $L(t)$ is the only length scale of the system. The scaling behavior of $L(t)$ implies the following ones for the kinetic energy and the enstrophy [3]: $\mathcal{K}=\left\langle v^{2}\right\rangle / 2 \sim t^{-2 / 3}$ and $\Omega=\left\langle\omega^{2}\right\rangle / 2 \sim t^{-5 / 3}(\omega=\nabla \times \boldsymbol{v}$ is the vorticity). Fig. 1 shows that the scaling predictions are well reproduced by our DNS. We remark that in the absence of stirring, phase separation is accelerated by the presence of hydrodynamics.

Stirred case. We now consider the presence of an external mechanical forcing acting on the velocity field. As customary in turbulent simulations, energy is injected by means of a random, time uncorrelated, homogeneous, and isotropic Gaussian process with amplitude $F$ which is restricted to a few Fourier modes around $k_{f}$ (this identifies the injection scale $\left.\ell_{f} \sim 2 \pi / k_{f}\right)$. The $\delta$-correlation
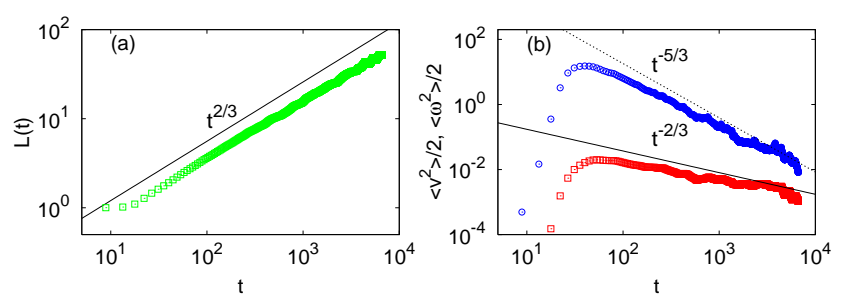

FIG. 1: (color online) (a) $L(t)$ vs $t$ obtained by DNS of (2) and (3) with $\xi=0.015, \nu=10^{-3}$, without external forcing. (b) Kinetic energy $\mathcal{K}=\left\langle v^{2}\right\rangle / 2$ (bottom) and enstrophy $\Omega=$ $\left\langle\omega^{2}\right\rangle / 2$ (top) vs $t$ in the same run.

in time allows for controlling the kinetic energy input $\epsilon_{i n}=F^{2} n_{f}$ (being $n_{f}$ the number of excited Fourier modes). Equations (2)-(3) are integrated starting with $\boldsymbol{v}=0$. In Fig. 2 we show typical snapshots of the order parameter at different flow intensities, and for two random forcing with $\ell_{f} \approx 26 \xi$ and $\ell_{f} \approx 84 \xi$. As it is evident, the stronger the stirring intensity the smaller the typical domain length. This is confirmed by the temporal evolution of $L(t)$ at varying the external forcing (Fig. 3). After an initial growth characterized by the $2 / 3$ scaling exponent, $L(t)$ stabilizes at a value $L^{*}$ that decreases with the stirring intensity. Both the kinetic energy $\mathcal{K}(t)$ and the enstrophy $\Omega(t)$ (not shown here) stabilize at corresponding values. Therefore a well defined statistically steady state is reached.

A closer inspection of Fig. 2 reveals qualitative differences in domain shapes. When $L^{*}$ is larger than the forcing scale $\ell_{f}$ (left) the domains are almost isotropic, while in the case $L^{*}<\ell_{f}$ (right) the underlying velocity field reveals itself through the filamental structure of the domains. Nevertheless, coarsening process is always arrested confirming the robustness of the phenomenon. Stirring always selects a scale through the competition between the thermodynamic forces and the stretching induced by local shears that deform and break the domains. Estimating the shear rate as $\gamma=u_{r m s} / L^{*}$ we find that,
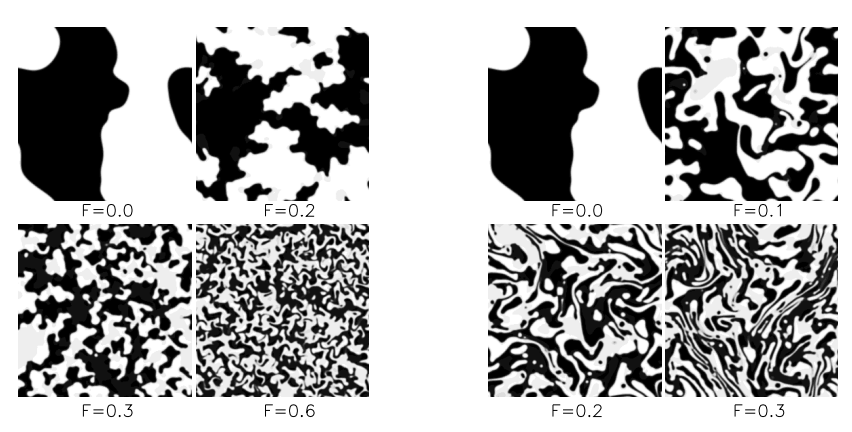

FIG. 2: Snapshots of $\theta$ at time $t=4000$ at varying the forcing intensity $F$ with $\ell_{f}=26 \xi$ (left) and $\ell_{f}=84 \xi$ (right). Black/white codes $\theta= \pm 1$. Other parameters are set as in Fig. 1 


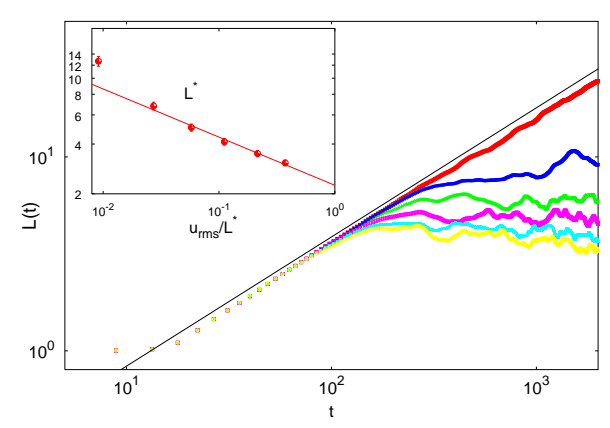

FIG. 3: (color online) $L$ vs $t$ at varying $F$, from top $F=0$ (thick curve) and $F=0.05,0.10,0.15, \ldots, 0.30$. Data refer to DNS with $\ell_{f}=84 \xi$ (the case with $\ell_{f}=26 \xi$ is qualitatively similar). The straight line displays the scaling $t^{2 / 3}$. Inset: $L^{*}$ vs $u_{r m s} / L^{*}$; the straight line has slope -0.29 , point size is of the order of the statistical error.

for the case with $L^{*}<\ell_{f}, L^{*} \sim \gamma^{-0.29}$ (see inset of Fig. 3), in fairly good agreement with experiments and simulations in pure shear flows [11]. However we should mention that in our settings, homogeneous and isotropic flows, there is not a well defined rate $\gamma$ as in genuine shear flows. The definition adopted here is a dimensional estimation of the shear rate at the arrest scale. In the case $L^{*} \geq \ell_{f}$ no clear scaling behavior is observed.

The existence of a stationary state can be understood in terms of conservation laws. Due to the presence of two inviscid quadratic invariants, $\mathcal{K}$ and $\Omega$, the single fluid $2 \mathrm{D}$ NS equation (i.e. (3) without the feedback term) is characterized by a double cascade [22]: $\mathcal{K}$ flows toward the large scales $\left(r>\ell_{f}\right)$ and $\Omega$ toward the small ones $\left(r<\ell_{f}\right)$. By switching on the coupling term, the following balance equation for the total energy $E=\mathcal{K}+\Phi$ holds [23]

$$
\frac{d E}{d t}=-\nu\left\langle|\nabla v|^{2}\right\rangle-\Gamma\left\langle|\nabla \mu|^{2}\right\rangle+\epsilon_{i n} .
$$

Now it is clear that in the unstirred case $\left(\epsilon_{i n}=0\right)$ asymptotically an equilibrium state, corresponding to fluid at rest $v=0$ and complete phase separation (minimum of free energy), is reached. In this case the velocity has only a transient role, determining the scaling of the coarsening process. On the contrary if $\epsilon_{i n} \neq 0$ a nontrivial stationary state stems from the balance of dissipative and input terms in the r.h.s. of (4).

It is worth mentioning that when the stirring intensity becomes high enough to overcome the feedback term, the kinetic energy dissipation induced by the $|\nabla \mu|^{2}$ term is no more effective. Indeed, when $\ell_{f}$ is much larger than $\xi$ and $F$ is very high, the coupling term becomes negligible and we observe the single-fluid phenomenology with an inverse energy cascade.

Passive binary mixtures We now consider the case in which the coupling term in (3) is switched off and consequently the order parameter is passively transported by the velocity field. This case has been already considered
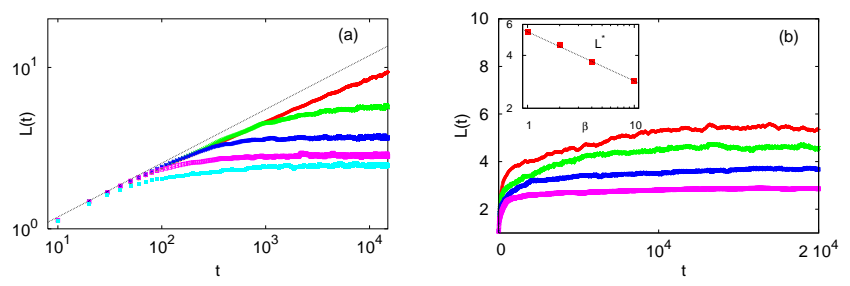

FIG. 4: (color online) Results of DNS in the passive case. (a) $L$ vs $t$ with (from top) $\beta=0.25,0.5,1,2,4$. Forcing parameters are $k_{f}=70$ and $F=3 \times 10^{-5}$. The straight line has slope $1 / 3$. (b) Same as (a), in linear scale, for a frozen velocity field (see text), from top $\beta=1,2,4,10$. Inset: $L^{*}$ vs $\beta$; the straight line corresponds to $L^{*} \sim \beta^{-0.28}$, point size is of the order of the statistical error. DNS were performed with hyper dissipation $-\nu_{2} \Delta^{2} \boldsymbol{v}$ with $\nu_{2}=10^{-7}$, and friction coefficient $\alpha=0.1$. The parameters of Eq. (2) are $\xi=0.015, \Gamma=0.02$.

in $[16,17]$. In order to obtain a statistically stationary state, as customary we added a large scale friction term $-\alpha \boldsymbol{v}$ to the Navier-Stokes equation [24]. The velocity field in (2) is rescaled by a factor $\beta$; this is a numerically convenient way to change the velocity intensity and to study the effect of stirring on coarsening. For $\beta=0$ Eq. (2) recovers the Cahn-Hilliard equation in a fluid at rest for which $L(t) \sim t^{1 / 3}$. For $\beta>0$ we observe the following phenomenology (Fig. 4a). For small values of $\beta$ (weak stirring) we did not find a clear evidence of coarsening arrest. This is likely due to finite size effects hiding the phenomenon, i.e. $L^{*}$ becomes comparable with, or even larger than, the box size. For $\beta$ large enough (strong stirring), the existence of an arrest scale $L^{*}$ (that decreases with $\beta$ ) is well evident.

Previous studies stressed the importance of Lagrangian Chaos in the coarsening arrest phenomenon [17]. Now, in order to elucidate this point, we discuss a non-chaotic example. It is well known (see, e.g., [18]) that two-dimensional stationary flows do not generate chaotic trajectories. We have thus integrated (2) in a frozen configuration of the turbulent velocity field: $\boldsymbol{v}(\boldsymbol{x}, t)=\boldsymbol{v}(\boldsymbol{x})$. As shown in Fig. 4b, domain growth is strongly weakened and finally arrested, even in this non-chaotic flow. For moderate velocity intensities, $L(t)$ still grows in time, but with a much slower scaling law than the dimensional prediction for fluids at rest, $t^{1 / 3}$. This slowing down is probably due to a different growth mechanism: after an initial transient, a slow process of droplet passage among close domains is indeed observed. However, for high enough intensities a complete stabilization of the domain length is realized. This suggests that the main ingredient for coarsening arrest is the presence of local shears that overwhelm the surface tension driving force. The dependence of $L^{*}$ on the shear rate (here naturally defined as $\beta$ ) is shown in the inset of Fig. 4b. We find a power law behavior with exponent -0.28 very close to the one observed in the 

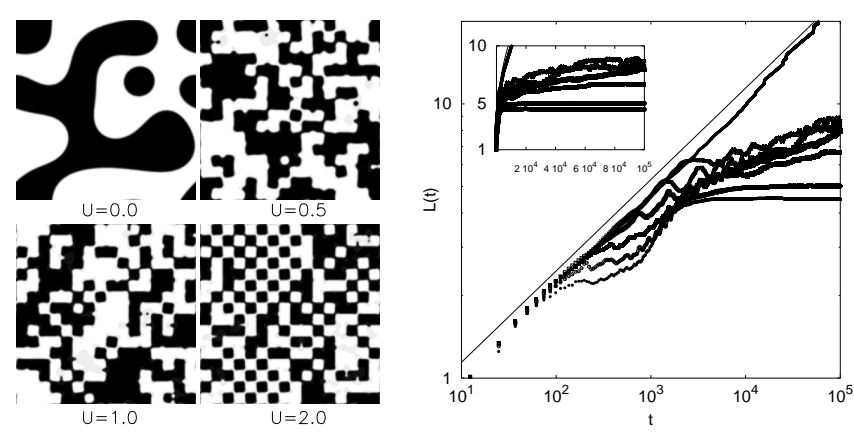

FIG. 5: (left) Snapshots of $\theta$ at $t=6 \times 10^{4}$ for the cellular flow with different $U$ and $\mathrm{K}=8$, here $\xi=0.018, \Gamma=0.1$. (right) $L$ vs $t$, from top to bottom $U=0,0.125,0.25,0.5,1.0,2.0,4.0$. Inset: the same in linear scale.

active case and in shear flows [11], while in the chaotic case no clear scaling is observed.

To further support the marginal role of Lagrangian Chaos in coarsening arrest, we report the results obtained in a stationary regular cellular flow $\left(v_{x}=U \sin (\mathrm{K} x) \cos (\mathrm{K} y), \quad v_{y}=-U \cos (\mathrm{K} x) \sin (\mathrm{K} y)\right)$ where $U$ fixes the velocity amplitude and $K$ the characteristic scale. As shown in Fig. 5 (left), for large intensities the order parameter is frozen into a random chessboard pattern with a finite length. At lower intensities a growth much slower than in the absence of the flow is still visible (Fig. 5 (right)) coming from a slow droplet migration from one cell to another. At large $U$ 's the shear between the counter-rotating vortexes overwhelms the demixing induced by the thermodynamic forces, breaking the domains which freeze into the cells.

In conclusion, we have shown that in the presence of an external stirring coarsening process is slowed down both for active and passive mixtures. We have also demonstrated that the phenomenon of coarsening arrest, first predicted in [6], does not necessarily require a chaotic flow, as suggested in [17], but is a consequence of the competition between thermodynamic forces and stretching induced by local shears. Our investigation in both active and passive mixtures shows that this behavior is robust. Moreover we found numerical evidence that the dependence of the arrest scale on the shear rate follows a power law behavior with an exponent close to the one measured in experiments and numerical simulations in pure shear flows [11]. Our results might suggest the existence of a mechanism independent of the nature of the flow in the coarsening arrest. Further numerical and experimental investigations, with the aim of clarifying the dependence of the arrest scale on the flow properties, would be extremely interesting.

We are grateful to A. Celani, G. Gonnella and S. Musacchio for useful discussions. We acknowledge partial support from MIUR Cofin2003 "Sistemi Complessi e Sistemi a Molti Corpi", and EU under the contract HPRN-CT-2002-00300. MC acknowledges the MPIPKS for computational resources.

[1] H. Furukawa, Adv. Phys. 43, 357 (1994); A. Bray, Adv. Phys. 34, 703 (1994).

[2] E.D. Siggia, Phys. Rev. A 20, 595 (1979).

[3] V.M. Kendon, M.E. Cates, I. Pagonabarraga, J.C. Desplat and P. Blandon, J. Fluid Mech. 440, 147 (2001).

[4] A.J. Wagner and J.M. Yeomans, Phys. Rev. Lett. 80, 1429 (1998); J.M. Yeomans, Ann. Rev. Comp. Phys. VII, ed. D. Stauffer, 61 (1999).

[5] A. Onuki, J. Phys: Condens. Matter 9, 6119 (1997).

[6] R. Ruiz and D.R. Nelson, Phys. Rev. A 23, 3224 (1981); J.A. Aronovitz and D.R. Nelson, Phys. Rev. A 29, 2012 (1984).

[7] A. Onuki, Phys. Lett. A 101, 286 (1984).

[8] M.E. Cates, V.M. Kendon, P. Bladon and J.C. Desplat, Faraday Discuss. 1121 (1999).

[9] F. Corberi, G. Gonnella and A. Lamura, Phys. Rev. Lett. 81, 3852 (1998); Phys. Rev. E 61, 6621 (2000).

[10] L. Berthier, Phys. Rev. E 63, 051503 (2001).

[11] T. Hashimoto, K. Matsuzaka, E. Moses and A. Onuki, Phys. Rev. Lett. 74, 126 (1995); Z. Shou and A. Chakrabarti, Phys. Rev. E 61, R2200 (2000).

[12] D.J. Pine, N. Easwar, J.V. Maher and W.I. Goldburg, Phys. Rev. A 29, 308 (1984).

[13] C.K. Chan, W.I. Goldburg and J.V. Maher, Phys. Rev. A 35, 1756 (1987); P. Tong, W.I. Goldburg, J. Stavans and A. Onuki, Phys. Rev. Lett. 62, 2668 (1989).

[14] K.Y. Min, J. Stavans, R. Piazza and W.I. Goldburg, Phys. Rev. Lett. 63, 1070 (1989); K.Y. Min and W.I. Goldburg, Phys. Rev. Lett. 70, 469 (1993).

[15] F. J. Muzzio, M. Tjahjadi, and J. M. Ottino, Phys. Rev. Lett. 67, 54 (1991).

[16] A.M. Lacasta, J.M. Sancho and F. Sagués, Phys. Rev. Lett. 75, 1791 (1995).

[17] L. Berthier, J.-L. Barrat and J. Kurchan, Phys. Rev. Lett. 86, 2014 (2001).

[18] A. Crisanti, M. Falcioni, G. Paladin and A. Vulpiani, Riv. Nuovo Cim. 14, 1 (1991).

[19] H. Tanaka, Phys. Rev. Lett. 72, 3690 (1994); H. Tanaka and T. Araki, Phys. Rev. Lett. 81, 389 (1998).

[20] G. Satten and D. Ronis, Phys. Rev. Lett. 55, 91 (1985).

[21] Measures based on the correlation function, $C(r, t)=$ $\langle\theta(\boldsymbol{x}, t) \theta(\boldsymbol{x}+\boldsymbol{r})\rangle, \quad$ or on the spherically averaged wavenumber weighted with the structure factor i.e. $L(t)=S\left[\int d k k^{q} S(k, t) / \int d k S(k, t)\right]^{-1 / q}$ (being $S(k, t)=$ $\left.\left\langle|\hat{\theta}(\boldsymbol{k}, t)|^{2}\right\rangle_{\mid \boldsymbol{k} \models k}\right)$ give equivalent results. See H. Furukawa, Phys. Rev. E 61, 1423 (2000) for a discussion on the different definitions. At large times, finite size effects spoil the scaling laws.

[22] R. Kraichnan, Phys. Fluids 10, 1417 (1967).

[23] Eq. (4) is obtained by averaging (2) and (3) after multiplication by $\mu$ and $\boldsymbol{v}$ respectively. The use of homogeneity, Gaussianity and $\delta$-correlation of the forcing gives the result.

[24] K. Nam, E. Ott, T.M. Antonsen, and P.N. Guzdar, Phys. Rev. Lett. 84, 5134 (2000). 